\title{
A influência de instrumentos de políticas públicas para a conservação - uma ótica sobre o pagamento dos serviços ambientais
}

\author{
The influence of public policies instruments for conservation - an overview about the payment of \\ environmental services
}

\author{
Mayara Bormann Azzulin 1 \\ Nicole Centurion ${ }^{2}$ \\ Niklas Werner Weins ${ }^{3}$ \\ Tatiana Maria Cecy Gadda ${ }^{4}$ \\ Carlos Mello Garcias ${ }^{5}$
}

\section{Resumo}

Os serviços ecossistêmicos são essenciais para a sobrevivência do ser humano. No entanto, devido ao processo de urbanização, a população perde a sensibilidade para com os recursos ambientais, trazendo como consequências impactos que podem se tornar irreversíveis. Ao tomar consciência da magnitude que esses impactos podem alcançar, o ser humano se vê na necessidade de alterar o entendimento acerca das cidades, desenvolvendo meios para o desenvolvimento sustentável. Atualmente, diversos instrumentos de gestão são utilizados para a conservação dos recursos ambientais, sendo um deles o pagamento por serviço ambiental (PSA). Assim, o objetivo deste trabalho foi levantar e compreender as contribuições do PSA para a configuração do território. A metodologia apresentou natureza exploratória, dividida nas etapas de busca bibliométrica e a análise dos artigos selecionados. Percebendo que se trata de um instrumento estratégico por envolver os três atores principais dentro da governança urbana (gestor público, população e o recurso natural), notou-se que existe uma hibridização na tipologia de instrumento de política ambiental, além de romper as barreiras entre o rural e urbano. Assim, o PSA passa a fortalecer as relações entre cidadãos e os serviços ecossistêmicos, sendo uma excelente resposta para as demandas das agendas globais atuais.

Palavras-chave: Pagamento por serviços ambientais. Urbano. Governança. Serviços ecossistêmicos.

\begin{abstract}
Ecosystem services are essential for human survival. However, due to the urbanization process, the population loses sensitivity to environmental resources, bringing as consequences impacts that may become irreversible. Human beings need to change their understanding about cities, becoming aware of the magnitude that these impacts can reach, and developing ways for sustainable development. Currently, several management instruments are used for the conservation of environmental resources, one of them is the payment for environmental service (PES). Thus, the objective of this study was to investigate and understand the contributions of the PES to the territory configuration. The methodology was based on exploratory research, divided into bibliometric search and analysis of selected articles. We noticed: a strategic instrument involving the three key actors within urban governance (public manager, population and natural resources); a hybridization in the typology of the environmental policy instrument; and breaking down barriers between rural and urban. Thus, PES strengthens relations between citizens and ecosystem services and is an excellent response to the demands of current global agendas.
\end{abstract}

Keywords: Payment for environmental services. Urban. Governance. Ecosystem services.

\footnotetext{
${ }^{1}$ Mestranda em Tecnologia e Sociedade pela UTFPR. E-mail: azzulin@alunos.utfpr.edu.br.

2 Mestranda em Gestão Urbana pela PUC/PR. E-mail: centurion.nicole@gmail.com.

${ }^{3}$ Doutorando pelo Núcleo de Estudos e Pesquisas Ambientais pela UNICAMP. E-mail: weinsniklas@gmail.com.

${ }^{4}$ Doutora em Earth and Human Environmental Science pela Chiba University. Professora no Programa de Pós-graduação em Tecnologia e Sociedade pela Universidade Tecnológica Federal do Paraná. E-mail: tatianagadda@utfpr.edu.br.

${ }^{5}$ Doutor em Engenharia Civil pela Universidade de São Paulo. Professor no Programa de Pós-graduação em Gestão Urbana da Pontifícia Universidade Católica do Paraná. E-mail: carlos.garcias@pucpr.br.
} 


\section{Introdução}

Um dos grandes questionamentos da atualidade nos diferentes âmbitos da governança é a relação entre urbanização e o meio ambiente. Com o aumento da população em áreas urbanas nas últimas décadas no mundo todo, elevou-se a demanda por serviços ecossistêmicos, tornando necessário o entendimento da relação dos impactos gerados pelo homem na natureza (WEST, 2017). Somado a isso, áreas naturais que anteriormente eram afastadas passam a se encontrar próximas às cidades (GÜNERALP; SETO, 2013), sendo necessária a preservação e o manejo adequado de tais áreas naturais, que provêm serviços ambientais (RECH; ALTMANN, 2009).

Ao longo dos últimos cinquenta anos, a visão de conservação da natureza vem se modificando. Segundo Mace (2014), inicialmente a preocupação com o meio ambiente estava voltada para conservação de espécies selvagens específicas/selecionadas em seus habitats, onde cada espécie permanecia dentro de um ecossistema. Na atualidade, a autora observa que a natureza soma ao ser humano, tornando homem e meio ambiente integrados em um ecossistema.

Nesse cenário surgem iniciativas globais que alteram o entendimento sobre cidades, que é o caso das agendas urbanas, como a Habitat 3, indicando a importância do desenvolvimento sustentável. Para que o almejo global reflita no nível local é necessária a implementação de políticas públicas voltadas ao ambiental e ao humano. Observando essa complexidade exigida no urbano, a boa governança é resultado da capacidade que o Estado tem de criar condições específicas e resolver os problemas locais de forma mais eficiente (KHAN, 2013).

Um dos exemplos de instrumentos importantes oriundos de iniciativas globais aplicadas no mundo inteiro é o Pagamento por Serviços Ambientais (PSA), que ajuda a fortalecer a comunicação sobre a importância dos Serviços Ambientais entre governo e sociedade (CASTRO-DÍAZ, 2014), e o entendimento da sociedade para com questões ambientais (TORRES et al., 2013) e até mesmo a valorização de práticas das comunidades que vivem em áreas rurais de forma monetária, em bens ou do reconhecimento delas (MOMBO et al., 2014).

A produção desses instrumentos de políticas públicas é formada no meio urbano e eles podem ser classificados como regulatórios voluntários e de cooperação, econômicos e de informação (MOURA, 2016), mas como as cidades são cada vez mais complexas (FREY, 2012), é normal a hibridização desses instrumentos (GUPTA et al., 2015). Desse modo, este trabalho visa analisar de que forma o PSA pode contribuir com o entendimento da configuração territorial dos indivíduos em áreas urbanas e rurais. 


\section{Referencial teórico}

A degradação a longo prazo dos ecossistemas pode ser evitada a partir do estabelecimento de um equilíbrio local entre o uso de recursos naturais e a população humana, denominado como o green loop. A modificação dos meios naturais exige, portanto, serviços não ecossistêmicos, que acabam acarretando mudanças nos sistemas de governança e institucional (CUMMING et al., 2014).

Frente à crescente exploração dos recursos naturais, houve a necessidade do estabelecimento de instrumentos para a racionalidade do seu uso, sendo inicialmente concebido sob o princípio do poluidor-pagador ou usuário-pagador, o qual determina que quem utiliza o recurso natural deve suportar seus custos (KFOURI; FAVERO, 2011). Atualmente, buscando uma nova atitude do homem em relação à natureza sob a perspectiva de que ela fornece serviços imprescindíveis para o bem-estar humano, necessitando de tempo e o espaço para prover tais serviços, surgem novos instrumentos como o Pagamento por Serviços Ambientais, o PSA (RECH; ALTMANN, 2009).

O PSA é um instrumento baseado no princípio provedor-recebedor, reconhecendo a importância do incentivo econômico àqueles que protegem as condições para os serviços oferecidos pelo meio ambiente (WUNDER et al., 2009; KFOURI; FAVERO, 2011). Assim, objetiva de maneira geral que determinados atores, sejam eles públicos ou privados, assumam práticas adequadas perante o meio ambiente, bem como o uso dos recursos naturais, de tal forma a garantir a manutenção e melhoria do funcionamento dos ecossistemas, assegurando concomitantemente o fornecimento dos serviços originários deles (FGB et al., 2017), apresentando como objetivos principais a conscientização dos beneficiários sobre a importância dos serviços ambientais e a valorização dos que contribuem diretamente para a sua conservação (RECH; ALTAMANN, 2009).

O PSA pode ser definido como "transações voluntárias entre usuários de serviços e provedores de serviços que estão condicionados às regras acordadas de gestão de recursos naturais para gerar serviços externos" (WUNDER, 2015). De maneira mais simplificada, a ideia básica do PSA é a de que os beneficiários dos serviços ambientais devem realizar pagamentos aos provedores desses serviços (MORAES, 2012). Dessa forma, sendo caracterizados como usuários/beneficiários de serviços aquelas pessoas, físicas ou jurídicas, públicas ou privadas, que se beneficiam dos serviços ambientais, podendo ser os próprios provedores ou terceiros (FGB et al., 2017). Já os provedores de serviços correspondem àqueles que adotam práticas de manejo, conservação e/ou restauração dos ecossistemas, consistindo em proprietários do recurso ambiental, geralmente rurais (MORAES, 2012). Estes, basicamente, devem cumprir uma função de, por exemplo, gerenciar os recursos naturais que controlam na área pela qual são responsáveis, respeitando as regras estabelecidas contratualmente (WUNDER, 2015). 
Logo, entendendo que tanto os beneficiários como os provedores podem ser pessoas físicas ou jurídicas, públicas ou privadas, existem diferentes arranjos para pagar e receber por serviços ambientais. Isso dependerá de quem são as partes envolvidas nessas transações, tendo como exemplos mais comuns de mecanismos para obtenção de recursos para esse sistema de incentivo à conservação ambiental; os via legislação, por meio do estabelecimento da estrutura legal específica, criando condições para a determinação dos esquemas de PSA, tendo como fonte de recursos os orçamentos, royalties ou outros recursos "carimbados"; e os via livre mercado, no qual ocorre o pagamento pelos serviços ambientais por "compradores” voluntários dos serviços ambientais, como por exemplo, usuários de água que visam garantir qualidade de água e regularização de vazões; ou aqueles que buscam compensar ou mitigar a sua "pegada hídrica" (KFOURI; FAVERO, 2011).

De acordo com Wunder et al. (2009), todos os PSA existentes envolvem serviços ambientais relacionados a uma ou mais das seguintes categorias distintas representadas pela: retenção ou captação de carbono; conservação de serviços hídricos; conservação da biodiversidade; e conservação de beleza cênica. No entanto, há algumas condições necessárias para o funcionamento e implementação do PSA, dentre elas estão as precondições econômica, cultural, institucional e informacional (WUNDER et al., 2009). A primeira se refere à necessidade da existência de uma externalidade (benefício) que vale a pena ser compensada ao provedor. Na segunda, a motivação do provedor é receber os pagamentos perante a prestação de serviços, devendo responder positivamente a esses incentivos econômicos. Na terceira, a motivação é a necessidade de estabelecer uma relação de confiança mínima entre usuários e provedores de serviços para que haja o cumprimento mútuo do contrato. A última diz respeito à necessidade de definição e mensuração dos serviços promovidos pelos provedores, bem como monitoramento de sua provisão e negociação de contratos (WUNDER et al., 2009).

Dessa forma, uma transação como a realizada no PSA somente faz sentido se ela proporcionar um maior fornecimento de serviços em comparação com um cenário hipotético sem o PSA, denominado como cenário linha de base, o qual apresenta como finalidade servir como referência para elaboração e levantamento de pressuposições que envolvem o fornecimento do serviço sob perspectiva futura (FGB et al., 2017).

Dentro do território brasileiro, o PSA é evidenciado principalmente em ações de proteção a bacias hidrográficas e manejo florestal (OUVERNEY et al., 2017), já que esses estão associados diretamente a certos riscos ambientais. Assim, o efetivo sucesso do PSA está diretamente relacionado com atores da região de interesse; as fontes alternativas de recursos financeiros; a capacidade institucional; o grau de mobilização social; e os critérios técnicos para indicação dos locais para conservação (FERNANDES; BOTELHO, 2016). 
Como exemplo de PSA para o manejo na Floresta Amazônica brasileira, há a experiência do "Programa de Desenvolvimento Socioambiental da Produção Familiar Rural (Proambiente)”, do Ministério do Meio Ambiente (FASIABEN et al., 2009), que funciona por meio de polos, onde é formado um conjunto de grupos responsáveis pela elaboração do "Acordo Comunitário", que com concordância com o Plano de Utilização da Unidade de Produção e o Padrão de Certificação de Serviços Ambientais, os produtores recebem o direito ao uso do selo do Proambiente como remuneração dos serviços ambientais. O efeito da renda acaba por se tornar um fator positivo para adesão (OUVERNEY et al., 2017).

Sob outra perspectiva, como as bacias hidrográficas são um recurso de interesse comum/público pelo seu uso, existe a necessidade da elaboração de alternativas de arranjos institucionais para o gerenciamento dos recursos hídricos. Esses arranjos envolvem a integração de pessoal capacitado, a constituição de instâncias que objetivam o comprometimento dos diferentes níveis de governo e da sociedade. É necessária a responsabilização de todos os atores envolvidos na gestão dos recursos hídricos para o desenvolvimento de políticas públicas de incentivos ambientais (ROSA; TONELLO; LOURENÇO, 2016).

Uma política pública, portanto, pode ser compreendida como um conjunto de formulações conceituais geradas por um ente governamental para possibilitar ações práticas destinadas à solução de determinadas questões que visam ao interesse público (FGB et al., 2017). Dentre estas questões, encontra-se o meio ambiente, que perante a Constituição Federal de 1988 em seu Capítulo VI e Artigo 225, é considerado um direito de todos tê-lo na condição ecologicamente equilibrada, sendo fundamental uma política que medeie os conflitos de uso dos recursos naturais, por meio de uma gama de instrumentos de naturezas regulatória, econômica, voluntária ou de informação (Quadro 1) para reduzir e/ou eliminar os problemas ambientais e alcançar o equilíbrio ambiental (MOURA, 2016).

QUADRO 1 - INSTRUMENTOS DE POLÍTICA AMBIENTAL

\begin{tabular}{|c|l|}
\hline Instrumentos & \multicolumn{1}{c|}{ Descrição } \\
\hline $\begin{array}{c}\text { Instrumentos } \\
\text { regulatórios }\end{array}$ & $\begin{array}{l}\text { Por meio de concessões ou proibições precedentemente estabelecidas, alicerçadas em restrições } \\
\text { legais, regulamentações ou normatizações, direciona-se o comportamento da coletividade. Estes } \\
\text { instrumentos impõem ação definida em relação ao meio ambiente, sendo caracterizados como } \\
\text { coercitivos por restringirem ou limitarem as opções perante as atividades econômicas. }\end{array}$ \\
\hline $\begin{array}{c}\text { Instrumentos } \\
\text { econômicos }\end{array}$ & $\begin{array}{l}\text { Apresentam como objetivos direcionar e incentivar comportamentos favoráveis ao meio } \\
\text { ambiente mediante benefícios ou custos referentes às alternativas de ação, baseando-se nos } \\
\text { princípios usuário-pagador ou protetor-recebedor. }\end{array}$ \\
\hline $\begin{array}{c}\text { Instrumentos } \\
\text { voluntários e de } \\
\text { cooperação }\end{array}$ & $\begin{array}{l}\text { Abrangem os diversos instrumentos de caráter voluntário e de cooperação entre os entes } \\
\text { envolvidos por meio de: contratos negociados, compromissos e acordos voluntários, } \\
\text { autorregulação voluntária e instrumentos de cooperação interinstitucional. }\end{array}$ \\
\hline
\end{tabular}




\begin{tabular}{|c|l|}
\hline $\begin{array}{c}\text { Instrumentos de } \\
\text { informação }\end{array}$ & $\begin{array}{l}\text { Por meio da disponibilização e disseminação de informações e valores favoráveis ao meio } \\
\text { ambiente, possuem como propósitos orientar, influenciar ou persuadir os agentes públicos ou } \\
\text { privados a agirem de maneira benéfica ante o meio ambiente, por meio da produção e } \\
\text { divulgação de dados referentes à qualidade ambiental, gestão ambiental, estudos, avaliações, } \\
\text { diagnósticos, materiais didáticos e conhecimento científico. }\end{array}$ \\
\hline
\end{tabular}

FONTE: Adaptado de MOURA (2016).

\section{Metodologia da pesquisa}

A primeira etapa do trabalho constituiu em uma pesquisa bibliométrica. Como estudos relacionados ao PSA são vistos no globo inteiro, optou-se por usar uma base de referências internacionais, a Web of Science (WoS). Esta apresenta um grande número de documentos que envolvem a área (FERNANDES; PHILIPPI JR., 2016). Já para compreender o estado da arte do PSA frente às políticas públicas das cidades brasileiras, tornou-se necessária a pesquisa na base de publicações científicas Scientific Electronic Library Online (Scielo), por ser uma biblioteca virtual voltada à produção científica da América Latina, com mais de $80 \%$ dos periódicos voltados para as ciências multidisciplinares e biológicas (PACKER, 2011).

O Quadro 2 sintetiza os diferentes grupos de palavras, tanto na Scielo quanto na WoS. Como as duas bases possuem idiomas principais diferentes, português e inglês, foi necessário modificar os grupos de palavras de busca, mas com o mesmo significado de tradução.

\section{QUADRO 2 - IDENTIFICAÇÃO DE ARTIGOS}

\begin{tabular}{|c|c|c|}
\hline \multicolumn{3}{|c|}{ Scientific Electronic Library Online (Scielo) } \\
\hline Grupo de palavras & Total de artigos & $\begin{array}{c}\text { Artigos } \\
\text { selecionados }\end{array}$ \\
\hline Pagamentos por serviços ambientais "and" urbano & 0 & 0 \\
\hline Pagamentos por serviços ambientais "and" rural & 4 & 2 \\
\hline PSA "and" urbano & 0 & 0 \\
\hline PSA “and” rural & 15 & 4 \\
\hline \multicolumn{3}{|c|}{ Web of Science (WoS) } \\
\hline Payment for environmental services "and" urban & 67 & 3 \\
\hline Payment for environmental services "and" rural & 232 & 4 \\
\hline PES “and” urbano & 82 & 3 \\
\hline PES “and” rural & 125 & 3 \\
\hline
\end{tabular}

FONTE: Os autores (2018).

Disposto de todos os trabalhos obtidos pela Scielo e WoS, primeiramente eles foram filtrados pela leitura dos títulos, e passada essa etapa foram lidos todos os resumos dos trabalhos remanescentes para obter o resultado dos artigos selecionados apresentados no Quadro 2.

A partir da leitura dos artigos selecionados, a segunda etapa do trabalho foi analisar de que forma existe a mudança na percepção territorial dos indivíduos que estão interagindo na região que 
abrange o PSA, como também os que não estão, mas que a partir de pagamentos e conscientização acabam por fazer parte.

\section{Resultados e discussão}

O PSA está entre os instrumentos criados com a finalidade de amenizar e controlar os impactos gerados sobre o meio ambiente devido ao processo de urbanização, trazendo incentivos que podem favorecer aqueles que colaborarem e respeitarem a legislação (CIDREIRA, 2016).

Dessa forma, notou-se que para sua implantação além de adequar o local às questões ambientais, identificando o modelo ideal de PSA junto com a definição dos parceiros e fontes financiadoras, é de extrema importância a instituição de um marco legal, realizando a promulgação de uma lei, seguido da regulamentação por meio de um decreto, isso para firmar um acordo entre todos os atores envolvidos, dado que as áreas previstas para a proteção como bacias hidrográficas e áreas florestais contemplam diferentes territórios (CIDREIRA, 2016).

O Quadro 3 demonstra como foram sistematizadas as análises sobre a literatura encontrada na revisão bibliométrica realizada nas bases selecionadas.

QUADRO 3 - RESULTADO DA REVISÃO BIBLIOMÉTRICA

\begin{tabular}{|c|c|c|c|}
\hline Tema & Objetivo & Instrumento & Fonte \\
\hline $\begin{array}{l}\text { PSA para questões } \\
\text { ambientais rurais }\end{array}$ & $\begin{array}{l}\text { Voltado à proteção fora do ambiente } \\
\text { urbano e que sofre pressão por } \\
\text { degradação e desmatamento }\end{array}$ & $\begin{array}{l}\text { Informação; } \\
\text { econômicos; } \\
\text { cooperação e acordos } \\
\text { voluntários. }\end{array}$ & $\begin{array}{l}\text { DA SILVA et al., 2017; } \\
\text { MOMBO } \text { et al., 2014; } \\
\text { TORRES } \text { et al., 2013; } \\
\text { OUVERNEY et al., 2017; } \\
\text { NARVAEZ R. } \text { et al., } 2016 .\end{array}$ \\
\hline $\begin{array}{l}\text { PSA para questões } \\
\text { ambientais urbanas }\end{array}$ & $\begin{array}{l}\text { Voltado ao manejo de elementos } \\
\text { naturais que possuam pouco } \\
\text { investimento e que fazem parte das } \\
\text { áreas urbanas }\end{array}$ & $\begin{array}{l}\text { Econômicos; } \\
\text { cooperação e acordos } \\
\text { voluntários; } \\
\text { informação. }\end{array}$ & $\begin{array}{l}\text { DA SILVA et al., } 2017 \text {; } \\
\text { MOMBO et al., 2014; } \\
\text { DAVIES et al., } 2018 .\end{array}$ \\
\hline $\begin{array}{l}\text { PSA relacionado às } \\
\text { comunidades que } \\
\text { possuem locais com } \\
\text { relevância ambiental }\end{array}$ & $\begin{array}{l}\text { Voltado à necessidade de } \\
\text { compreensão das dinâmicas de } \\
\text { degradação ambiental e da realidade } \\
\text { sobre a situação da população que } \\
\text { vive nesses ambientes }\end{array}$ & $\begin{array}{l}\text { Econômicos; } \\
\text { cooperação e acordos } \\
\text { voluntários; } \\
\text { regulatórios. }\end{array}$ & $\begin{array}{l}\text { FIGUEROA et al., 2016; } \\
\text { ARRIAGADA } \text { et al., } \\
\text { 2015; } \\
\text { NIERATKAA; BRAY; } \\
\text { MOZUMDER, 2015; } \\
\text { FASIABEN } \text { et al., 2009. }\end{array}$ \\
\hline $\begin{array}{l}\text { PSA voltado a } \\
\text { questões hídricas }\end{array}$ & $\begin{array}{l}\text { Voltado à proteção de mananciais de } \\
\text { abastecimento público, na qual o } \\
\text { principal ator envolvido é o } \\
\text { proprietário rural }\end{array}$ & $\begin{array}{l}\text { Informação; } \\
\text { econômicos; } \\
\text { cooperação e acordos } \\
\text { voluntários. }\end{array}$ & $\begin{array}{l}\text { NARVAEZ R. et al., } \\
2016 ; \\
\text { CASTRO-DÍAZ, 2014; } \\
\text { OUVERNEY et al., 2017; } \\
\text { LIBANIO, 2016; } \\
\text { JARDIM; BURSZTYN, } \\
2015 \text {. }\end{array}$ \\
\hline
\end{tabular}

FONTE: Os autores (2018).

A grande maioria dos artigos relaciona a área rural ao PSA e artigos que são voltados às questões hídricas também estão fora do ambiente urbano. Todos os artigos encontrados relacionam a 
política pública como instrumento econômico. A questão monetária acaba sendo importante devido à capacitação e destinação de recursos para a implementação e efetivação do serviço ecossistêmico (NARVAEZ R. et al., 2016), mesmo que as probabilidades sejam variáveis conforme a localização geográfica (OUVERNEY et al., 2017).

Mas nem sempre a melhor maneira de efetivar o PSA é a financeira, já que existem benefícios não monetários, como melhorias nos serviços de saúde e educação, bem como a geração de emprego, que podem oferecer uma melhor integração e participação da sociedade com o PSA (TORRES et al., 2013).

Destaca-se aqui o PSA ligado a questões urbanas no que tange ao instrumento ser informativo, já que muitas vezes por razões de marketing verde as empresas tendem a divulgar que estão participando, de alguma forma, para ajudar que as áreas urbanas sejam mais sustentáveis (DAVIES et al., 2018). Iniciativas público-privadas acabam por se integrar a partir da formalização de acordos e garantir uma relação mais equilibrada, estabelecendo cooperação não só entre empresa e governo, mas também em âmbitos regionais, como é o caso do PSA voltado para recursos hídricos (LIBANIO, 2016), que é um dos exemplos mais discutidos atualmente para aplicação do PSA.

Para o PSA voltado às comunidades, existem dois contrapontos: o primeiro que estabelece um efeito positivo, já que pode vir a auxiliar de forma monetária comunidades que normalmente são carentes (NIERATKAA; BRAY; MOZUMDER, 2015); e que por mais que exista a percepção pela comunidade urbana sobre as comunidades relacionadas às áreas dos PSA, os indivíduos dentro das áreas rurais não relacionam a política como de fato efetiva (MOMBO et al., 2014).

Das informações coletadas, observa-se que também são necessários arranjos institucionais para estabelecer de forma clara os responsáveis pelo gerenciamento do programa, sendo esta uma oportunidade para o desenvolvimento. A exemplo disso, tem-se a governança multinível, que consiste no envolvimento entre atores públicos e privados, gerando mecanismos de articulação que visam à integração de esforços horizontais e verticais para viabilizar o fornecimento de serviços de natureza pública, bem como uma paradiplomacia para envolver atores da esfera internacional para viabilizar questões econômicas, políticas e de cooperação (PROCOPIUCK, 2013).

\section{Considerações finais}

A crescente concentração da população mundial em áreas urbanas fez com que se exigisse mais dos serviços ecossistêmicos. Apesar do pensamento sobre conservação estar se modificando de forma positiva ao longo das décadas, reforça-se a necessidade do entendimento da relação entre homem e natureza, que com o fenômeno da urbanização tende a afastar essa interação. 
No começo dos estudos sociais, as questões urbanas e rurais eram vistas como opostas (REDFIELD, 1956), porém as iniciativas globais, que cada vez mais relacionam a importância do meio natural, como é o caso da Agenda 21, indicam que é necessário o equilíbrio da sustentabilidade através da realidade urbana por meio das políticas públicas, a fim de garantir a consciência sobre as questões ambientais (MEDAUAR; ALMEIDA, 2002).

Uma das formas de garantir a conscientização do homem sobre o meio ambiente e ao mesmo tempo manter a conservação deste último é o PSA. Esse instrumento de política pública ainda é pouco reconhecido no território brasileiro, mas acaba sendo estratégico por envolver os três elementos principais dentro da governança urbana: o gestor público, a população e recurso natural.

Foi possível observar que a maioria das iniciativas voltadas ao PSA é para o meio rural, mas além dessas políticas públicas serem produzidas no meio urbano, notou-se uma conscientização não só em relação ao meio natural, mas também com às comunidades que vivem nessas áreas, mesmo que estas ainda não tenham a consciência dessa importância. Assim, é possível concluir que existe um processo de rompimento entre as barreiras rurais e urbanas das comunidades que vivem principalmente nas cidades.

Como o instrumento tenta resolver questões de diferentes posições geográficas, este acaba por ser moldável, o que resulta em uma hibridização na tipologia dos instrumentos, bem como na variação destes que são: regulatórios, econômicos, informativos e de cooperação. Apesar da degradação ambiental ser inevitável, o PSA, além de conscientizar a população que está compreendida no recorte territorial sobre o instrumento, passa a assegurar e fortalecer a relação não só do cidadão e meio natural, mas também a relação de cidadão e dos seus gestores públicos.

\section{Agradecimentos}

O presente trabalho foi realizado com apoio da Coordenação de Aperfeiçoamento de Pessoal de Nível Superior - Brasil (CAPES) - Código de Financiamento 001.

\section{Referências}

ARRIAGADA, R. A.; SILLS, E. O.; FERRARO, P. J.; PATTANAYAK, S. K. Do payments pay off? Evidence from participation in Costa Rica's PES Program. PloS one, v. 10, n. 8, p. e0136809, 2015.

CASTRO-DÍAZ, R. Implicancias territoriales de los esquemas de pago por servicios ambientales (PSA) en cuencas norandinas. Cuadernos de Geografía - Revista Colombiana de Geografía, Bogotá, v. 23, n. 1, p. 61-74, 2014. 
CIDREIRA, L. E. Pagamentos por serviços ambientais como instrumento para a gestão das águas em ambientes urbanos. 2016. 134f. Dissertação de Mestrado (Programa de Pós-Graduação em Gestão Urbana) - Pontifícia Universidade Católica do Paraná, Curitiba, 2016.

CUMMING, G. S.; BUERKERT, A.; HOFFMANN, E. M.; SCHLECHT, E.; VON CRAMON-TAUBADEL, S.; TSCHARNTKE, T. Implications of agricultural transitions and urbanization for ecosystem services. Nature, v. 515, p. 50-57, 2014.

DA SILVA, R. F. B.; RODRIGUES, M. D. A.; VIEIRA, S. A.; BATISTELLA, M.; FARINACI, J. Perspectives for environmental conservation and ecosystem services on coupled rural-urban systems. Perspectives in Ecology and Conservation, v. 15, n. 2, p. 74-81, 2017.

DAVIES, H. J.; DOICK, K. J.; HUDSON, M. D.; SCHAAFSMA, M.; SCHRECKENBERG, K.; VALATIN, G. Business attitudes towards funding ecosystem services provided by urban forests. Ecosystem services, v. 32, p. 159-169, 2018.

FASIABEN, M. do C. R.; ANDRADE, D. C.; REYDON, B. P.; GARCIA, J. R.; ROMEIRO, A. R. Estimativa de aporte de recursos para um sistema de pagamento por serviços ambientais na Floresta Amazônica Brasileira. Ambiente \& Sociedade, v. 12, n. 2, p. 223-239, 2009.

FERNANDES, V.; PHILIPPI JR., A. Sustainability Sciences. The Oxford Handbook of Interdisciplinarity, p. 370, 2017.

FERNANDES, L. S.; BOTELHO, R. G. M. Proposta Metodológica de Priorização de Municípios para Implantação de Programas de Pagamento por Serviços Ambientais (PSA). Ambiente \& Sociedade, São Paulo, v. 19, n. 4, p. 101-120, 2016.

FGB - Fundação Grupo Boticário de Proteção à Natureza; TNC - The Nature Conservancy do Brasil; MMA - Ministério do Meio Ambiente; GIZ - Deutsche Gesellschaft für Internationale Zusammenarbeit. Guia para a formulação de políticas públicas estaduais e municipais de pagamentos por serviços ambientais. 2017. 77p. Disponível em: <http://www.mma.gov.br/publicacoes/biodiversidade/category/143-economia-dos-ecossistemas-eda-biodiversidade>.

FIGUEROA， F.; CARO-BORRERO, A.; REVOLLO-FERNÁNDEZ， D.; MERINO, L.; ALMEIDA-LEÑERO, L.; PARÉ, L.; ESPINOSA, D.; MAZARI-HIRIART, M. "I like to conserve the forest, but I also like the cash". Socioeconomic factors influencing the motivation to be engaged in the Mexican Payment for Environmental Services Programme. Journal of Forest Economics, v. 22, p. 36-51, 2016.

FREY, K. Abordagens de governança em áreas metropolitanas da América Latina: avanços e entraves. Urbe - Revista Brasileira de Gestão Urbana, v. 4, n. 1, p. 87-102, 2012.

GUPTA, J.; PFEFFER, K.; ROS-TONEN, M.; VERREST, H. Setting the scene: The geographies of urban governance. In: Geographies of Urban Governance. Springer, Cham, p. 3-25, 2015.

GÜNERALP, B.; SETO, K. C. Futures of global urban expansion: uncertainties and implications for biodiversity conservation. Environmental Research Letters, v. 8, n. 1, p. 014025, 2013.

JARDIM, M. H.; BURSZTYN, M. A. Pagamento por serviços ambientais na gestão de recursos hídricos: o caso de Extrema (MG). Engenharia Sanitária Ambiental, v. 20, n. 3, p. 353-360, 2015. 
KFOURI, A.; FAVERO, F. Projeto conservador das águas passo a passo: uma descrição didática sobre o desenvolvimento da primeira experiência de pagamento por uma prefeitura municipal no Brasil. Brasília, DF: The Nature Conservancy do Brasil, 2011.

KHAN, M. H. Governance and development: the perspective of growth-enhancing governance. Eastern and western ideas for african growth: diversity and complementarity in development aid, p. $85-120,2013$.

LIBANIO, P. A. C. O uso de estratégias focadas em resultados para o controle da poluição hídrica no Brasil. Engenharia Sanitária Ambiental, v. 21, n. 4, p. 731-738, 2016.

MACE, G. M. Whose conservation? Science, v. 345, n. 6204, p. 1558-1560, 2014.

MEDAUAR, O.; ALMEIDA, F. D. M. de. Estatuto da cidade: Lei 10.257, de 10/07/2001, comentários. São Paulo: Revista dos Tribunais, 2002.

MOMBO, F.; LUSAMBO, L.; SPEELMAN, S.; BUYSSE, J.; MUNISHI, P.; HUYLENBROECK, G. Scope for introducing payments for ecosystem services as a strategy to reduce deforestation in the Kilombero wetlands catchment area. Forest Policy and Economics, v. 38, p. 81-89, 2014.

MORAES, J. L. A. Pagamento por Serviços Ambientais (PSA) como Instrumento de Política de Desenvolvimento Sustentável dos Territórios Rurais: o Projeto Protetor Das Águas de Vera Cruz, RS. Sustentabilidade em Debate, v. 3, n. 1, p. 43-56, 2012.

MOURA, A. M. M. de (Org.). Governança ambiental no Brasil: instituições, atores e políticas públicas. Brasília, DF: IPEA, 2016.

NARVAEZ, R. Y. A.; PAZ, A. W. J.; GUAPUCAL, C. M. del R.; LEONEL, H. F. Procesos institucionales en el pago por servicios ambientales, cuenca alta del río Pasto. Revista de Ciências Agrícolas, San Juan de Pasto, v. 33, n. 1, p. 64-72, 2016.

NIERATKAA, L. R.; BRAY, D. B.; MOZUMDER, P. Can payments for environmental services strengthen social capital, encourage distributional equity, and reduce poverty? Conservation and Society, v. 13, n. 4, p. 345-355, 2015.

OUVERNEY, I. R.; MOTTA, R. S. da; ORTIZ, R. A.; COELHO, P. S. Condicionantes da disposição de participar e aceitar pagamentos por serviços ambientais: um estudo de caso no Brasil. Revista de Economia Contemporânea, v. 21, n. 3, p. 1-27, 2017.

PACKER, A. L. Os periódicos brasileiros e a comunicação da pesquisa nacional. Revista USP, São Paulo, n. 89, p. 26-61, 2011.

PROCOPIUCK, M. Políticas públicas e fundamentos da administração pública: análise e avaliação, governança e redes de políticas, administração judiciária. São Paulo: Atlas, 2013.

RECH, A. U.; ALTMANN, A. Pagamento por serviços ambientais: imperativos jurídicos e ecológicos para preservação e a restauração das matas ciliares. Caxias do Sul, RS: EDUCS, 2009.

REDFIELD, R. Peasant society and culture: an anthropological approach to civilization. Chicago: University of Chicago Press, 1956. 
ROSA, F. S.; TONELLO, K. C.; LOURENÇO, R. W. Eleição de áreas prioritárias para pagamento por serviços ambientais: uma análise em nível de microbacia. Ambiente \& Água - An Interdisciplinary Journal of Applied Science, Taubaté, v. 11, n. 2, p. 448-461, 2016.

TORRES, A. B.; MACMILLAN, D. C.; SKUTSCH, M.; LOVETT, J. C. Payments for ecosystem services and rural development: landowner's preferences and potential participation in western Mexico. Ecosystem Services, v. 6, p. 72-81, 2013.

WUNDER, S.; BÖRNER, J.; TITO, M. R.; PEREIRA, L. Pagamentos por serviços ambientais: perspectivas para a Amazônia Legal. 2. ed. Brasília, DF: MMA, 2009.

WUNDER, S. Revisiting the concept of payments for environmental services. Ecological Economics, v. 117, p. 234-243, 2015.

WEST, Geoffrey B. Scale: the universal laws of growth, innovation, sustainability, and the pace of life in organisms, cities, economies, and companies. London: Penguin, 2017.

Artigo recebido em 29/11/2019. Aceito para publicação em 17/12/2019. 